\title{
Precautions adopted in a clinical chemistry laboratory as a result of an outbreak of serum hepatitis affecting hospital staff
}

\author{
I. W. PERCY-ROBB, J. PROFFITT, AND L. G. WHITBY \\ From the Department of Clinical Chemistry, The Royal Infirmary, Edinburgh
}

SYNOPSIS This article describes the precautions which have been introduced into a clinical chemistry laboratory with a view to reducing the risk of infection of staff with the virus of serum hepatitis.

The Royal Infirmary of Edinburgh has a chronic renal dialysis unit, and it has been recognized for several years that staff working in this type of unit are at particular risk from infection with the virus that causes serum hepatitis (New England J. Med., 1970). In 1969 a patient who was being maintained in Edinburgh on chronic renal dialysis developed serum hepatitis, and several other patients have since become infected. From the point of view of the hospital staff, this outbreak took a much more serious turn early in 1970 when a doctor contracted serum hepatitis and died. There have since been three other deaths among staff (a second doctor, a laboratory technician, and a laboratory clerk/receptionist) and some other members of staff have contracted a less severe form of the illness. It is not the purpose of this paper to discuss whether all these cases of serum hepatitis among staff form part of a linked pattern of infection-the epidemiological aspects of this outbreak have been studied extensively by our bacteriological colleagues, and their findings will be the subject of a separate report.

This outbreak of serum hepatitis associated with deaths among hospital staff prompted an urgent and careful review of the standards of practice in all those departments which might be responsible for carrying out work in relation to patients from whom infected samples might be obtained, whether or not these patients were overt cases of serum hepatitis. The potential seriousness of the danger was evident from the report of a
Swedish outbreak (Nyström and Ringertz, 1967) where 20 members of staff in the clinical chemistry department had been affected, although it should be stated that the incidence of serum hepatitis among laboratory workers reported in other outbreaks affecting hospital staff has been much lower.

The general review of preventive aspects contained in the report of a working party set up by the Public Health Laboratory Service (1968) served as a useful preliminary to the more detailed review of laboratory procedures. This review involved us in several discussions with the bacteriologists before a code of practice was drawn up. Copies of this code were provided to all members of staff, following a general explanatory talk, and copies are issued with a detailed explanation to new members of staff when they first report for work. We do not claim that this code of practice contains much that is original, but we have had several enquiries from the heads of clinical chemistry laboratories elsewhere in the United Kingdom, and they seem to have been grateful for being provided with copies of the precautions we have adopted. This widespread interest in an important and worrying problem constitutes our justification for publishing this report.

\section{Differences between Work of Clinical Chemists and of Bacteriologists}

The work of a clinical chemistry laboratory 
differs in several respects from the work of a bacteriology department, and these differences make it difficult to adopt in full the aseptic notouch techniques of the bacteriologists. Whereas much of the bacteriologist's work is concerned with specimens of sputum, urine, or faeces, all of which are to some extent unpleasant to deal with, most of the clinical chemist's work is to analyse specimens of blood. Attempts to educate laboratory workers to regard all specimens of blood as potentially dangerous, and to be handled at their peril, lose much of their impact when it is remembered that few diseases are transmitted by contact with blood and that the incidence of serious disease such as serum hepatitis, although a recognized hazard of laboratory work in hospitals, is undoubtedly low.

There is another important and relevant difference between much of the work of clinical chemists and of bacteriologists. For the most part, the staff in clinical chemistry departments are required to perform precise quantitative determinations, whereas much of the work of the bacteriologists is of a semi-quantitative or qualitative nature. It is difficult to obtain precise results, especially when these are to be based on determinations that require the accurate measurement of small volumes of viscous material such as plasma or serum, if the various analytical procedures have to be carried out by relatively remote control, using aseptic no-touch techniques.

The purpose of the review of laboratory procedure which we conducted earlier this year was to see to what extent the principles that govern the bacteriologist's approach to his laboratory work could be transferred and applied in a clinical chemistry department. In the precautions we have assumed that infection can be transferred by the faecal-oral route as well as via a break in the skin's surface or through the conjunctiva. One guiding principle that has had to be borne in mind in drawing up these precautions has been the one of practicability, since the rarity of serum hepatitis among laboratory staff in the past might otherwise tend to encourage non-observance of precautions which tended to slow down the output of work in the department. The overall effect of the precautions can perhaps be summarized by saying that they draw attention to the importance of personal hygiene, but there are points of detail which extend more widely.

\section{General Points on Laboratory Hygiene}

The first group of measures taken has been to reiterate the basic tenets of laboratory hygiene and cleanliness of operation, to tighten up the procedure for the reporting of accidents, and to appoint a departmental safety officer.

The importance of keeping laboratory working areas and passages clean and tidy has been emphasized, as has the need to dispose of litter $\frac{\rho}{\overline{5}}$ into waste buckets or (as detailed below) into redo plastic bags. The departmental cleaners have been instructed not to touch the benches or the analytical equipment or specimens of any kind $\overrightarrow{\overrightarrow{\vec{p}}}$ and the department's general tidiness therefore? depends greatly on the various members of staff?

All laboratory staff and visitors to the depart $\frac{\bar{\sigma}}{\bar{c}}$ ment are now required to wear white laboratory coats when in any part of the laboratory where analytical work is carried out, whether or notes they are handling specimens. These coats have to be removed before going for tea breaks in thedepartment or for meals elsewhere in the Royakw Infirmary. Staff are advised to wash their hands after using the lavatory or before going for meals? and it is considered that the act of removing whitew coats is an essentially practical reminder of thise basic hygienic requirement. An embargo has beener placed on taking food into the analytical working areas and related circulation space, and neithereating nor smoking is permitted in these parts $\overrightarrow{-}$ of the laboratory.

In addition to the wearing of white coats, when preparing samples of biological material (blood, $\bar{\partial}$ serum, plasma, urine, faeces, etc) all staff have $\stackrel{\mathbb{\Phi}}{-}$ been instructed to wear plastic aprons (details of $\overrightarrow{\overrightarrow{0}}$ suppliers, costs, etc, for these and several other items mentioned in this report are given in the Appendix) as well as their laboratory coats, ando only to handle these samples when wearing gloves. These instructions apply to the handling of allo specimens, and not merely to those designated as $\frac{0}{\circ}$ 'high risk' specimens (see below). Staff wearing gloves are instructed to apply barrier cream too랄 their hands before putting on gloves, to discard 3 the gloves after use, and to wash their hands and use hand cream after removing the gloves. Further, the timing of tea breaks and meal timeso has been so arranged that no one has to wear:gloves for more than two hours at a time. For 3 . staff working in the specimen reception area, $\stackrel{\circ}{\circ}$ these precautions mean that four pairs of gloves are used each day. The gloves chosen for generalo laboratory use fit loosely and have not given rise to dermatitis; however, if they do developo dermatitis at work, members of the staff have been instructed to report this so that their duties or or conditions of work can be adjusted.

It was already standard practice for accidents at work to be reported and recorded. With serum hepatitis as a possible consequence, however, careful consideration had to be given to the $\frac{C}{\Phi}$ question whether or not to administer $\gamma$-globulin. $\stackrel{\mathbb{Q}}{\rightarrow}$ The evidence for the value of $\gamma$-globulin in 0 preventing or modifying the course of serum hepatitis is conflicting, and it has been suggested $\stackrel{\odot}{\odot}$ that this may be due to differences in the virulence $\mathbb{\mathbb { D }}$ of various strains of the virus responsible for serum $\frac{\varrho}{\sigma}$ hepatitis (Lancet, 1970). There have also been reports indicating that the administration of 8 $\gamma$-globulin in itself may not be entirely free from risk (Allen and Kunkel, 1966; Vyas, Perkins, and 
Fudenberg, 1968). This aspect of our precautions in particular is being kept under constant review, and the procedure presently adopted may be changed. However, as it may serve some protective function (Krugman and Giles, 1970), at present we advocate the administration of $\gamma$-globulin ( $20 \mathrm{ml}$ intramuscularly, protein content, $750 \mathrm{mg} / 5 \mathrm{ml}$ ) to members of staff who, when handling high-risk specimens (as defined below) do one of the following:

(a) Cut themselves at work, if there was any possibility that blood, plasma, etc, from a patient might have entered the cut.

(b) Get blood, plasma, etc, in the mouth, despite the prohibition of mouth pipetting (see below).

(c) Get splashed in the eyes with blood, plasma, etc.

A departmental safety officer was appointed and a system of deputies arranged. The safety officer is a senior II technician who holds an AIMLT certificate in bacteriology in addition to other qualifications, and his main tasks as safety officer have been to supervize the training of staff in the precautions adopted, and to ensure the maintenance of the precautions thereafter. To assist the safety officer in this work, the Department of Bacteriology arranged a three-day introductory training course on bacteriological aspects of hospital laboratory work last August, about the time that a number of school leavers joined the staff of this and other hospital laboratory departments in Edinburgh.

Collection of Specimens, Sample Reception, and Preparation

The general points detailed here have been introduced with the intention that contact with samples of blood, plasma, urine, etc, be avoided at all times in the laboratory during the preparation of samples for analysis, during the actual processes of analysis, and at the time of disposal of residues or cleaning of apparatus. We also had to consider the safety of ward orderlies, porters, and others who transport specimens to the laboratory from many different hospitals and collection points.

All samples of blood, plasma, or serum have to be sent to the laboratory in plastic bags. Urine collections are sent in Winchesters provided with leak-proof rubber or plasticized rubber replacements of the top washers, and specimens of faeces are collected into waxed Mono containers and transported in plastic bags. When any of these specimens has been spilt, including spilling inside plastic bags, samples may be discarded at the discretion of the safety officer or his deputy. The onus on supplying and delivering specimens to the laboratory in a satisfactory state is thus placed on the users of the laboratory service.
Arrangements for High-risk Specimens

All specimens are regarded as potentially dangerous. However, steps have been taken to identify those specimens which should be treated as being particularly dangerous, in terms of the risk or possible risk to staff of transmitting serumE hepatitis. High-risk specimens, in this context $\frac{\overline{\bar{n}}}{\bar{p}}$ have been defined as specimens collected from jaundiced patients in whom the cause of the jaundice remains undiagnosed, from patientsึ suspected of having hepatitis, from rena $\vec{b}$ transplant cases, and from patients on chronicrenal dialysis. High-risk specimens are transportedw in plastic bags specially identified by the printing of '? hepatitis' in yellow letters on the bag. This allows these specimens to be singled out forw separate handling in batches in a high-risk sample preparation area. Forms accompanying sampleser from these patients are marked with a red star $\overrightarrow{0}$ As an additional safeguard, the safety officer, the duty clinical chemist, and the duty senior $\vec{\square}$ technician each has a list of names for thosed patients in whom positive results for Australia antigen tests have been obtained; these particu $\frac{3}{5}$ larly dangerous specimens are then additionally marked with the letters $\mathbf{A A}$ on arrival in the laboratory.

It used to be common practice to staple request forms to plastic bags to help to interrelate theme with their corresponding specimens. This practices is no longer allowed. Instead, request forms accompany specimens by being placed inside the्ठ plastic bags or more often are attached to the bag with adhesive tape. As far as the forms cor 0 responding to high-risk specimens are concerned, after the specimen has been removed from the bag, the request form (which is a combined. request and report form) is heated at $120^{\circ}-150^{\circ}$ कृ for $30 \mathrm{~min}$ in a hot air oven. The form is then' photocopied and the original destroyed.

One ready means of disseminating infection arises in the centrifuging of specimens of blood before the separation of plasma or serum. Aerosolso may form, particularly if a tube fractures during centrifugation. The possibility of venting centris fuges outside the laboratory has been discussect with the manufacturers and is said to be imer practicable. In the long term, therefore, it is planned to arrange for all centrifuges required for preparing samples to be situated under fume cupboard hood. For the present, alt specimens of blood are centrifuged with the tube $\$$ capped, and a separate centrifuge fitted with wind shield is reserved for the preparation ofo high-risk samples; this is situated in a separate fume cupboard supplied with its own direct exhaust system of ventilation. The same fumed cupboard is used for homogenizing any specimens్ of faeces.

Separation of plasma and serum from erythro응 cytes is carried out using Pasteur pipettes, an samples from high-risk specimens are identifie 
by being closed with a red cap after separation. Staff have been instructed to ensure that caps are removed carefully from the specimen tubes before aspiration of the plasma or serum-snapping off the caps can generate a fine spray of liquid. They have also been shown how to use the Pasteur pipettes so that there is no blowing of bubbles of plasma or serum due to careless squeezing of the rubber teat. In addition, the importance of using rubber teats with a volume less than the Pasteur pipette has been emphasized. After use, the pipettes are disposed of directly into $10 \%$ Chloros, by complete submersion. Laying Pasteur pipettes down on the bench, with the possibility that serum or plasma might run up into the teat, has been eliminated; pipettes are at all times held vertically when in use. We have not instituted the practice of wearing safety spectacles or masks at this stage, except insofar as these are now worn routinely when handling the high-risk specimens of blood, plasma, and serum, as well as when homogenizing any specimen of faeces.

\section{Laboratory Benches and Analytical Work}

Any area of bench that is used for the preparation of samples is covered with Benchkote. This applies also to areas used for the loading of AutoAnalyzer (Technicon Instruments Co., Chertsey, Surrey) sample plates, and for areas on which AutoAnalyzer sampler units and pumps are placed. Chloros $(10 \%)$ is always available where samples are prepared and plates are loaded, and the instructions are that any spillages are to be washed down immediately. The Benchkote is changed routinely once a week and the parts of the bench covered by Benchkote are swabbed with $10 \%$ Chloros at that time.

Pasteur pipettes are used as described already when loading cups on AutoAnalyzer plates before analysis. As previously indicated, gloves are worn when handling specimen tubes at the time of loading AutoAnalyzer plates or sampling for other purposes. As far as high-risk specimens are concerned, these samples are loaded on to separate AutoAnalyzer sampler plates and are analysed at the end of a run unless considerations of urgency dictate otherwise. For specimens analysed by procedures other than AutoAnalyzer methods, when taking a measured portion of a specimen of the biological material for analysis, staff again wear disposable gloves and it must be emphasized again that no pipetting of samples by mouth suction is allowed. Any excess of sample is wiped from the outside of the equipment used for drawing up the sample using disposable absorbent material, and this is discarded into the waste bin before the gloves are finally removed. The exception to this rule relates to the use of pipettes that have been plugged and fitted with a trap, this type of pipetting only being applicable for volumes of $100 \mu \mathrm{l}$ or less. For the most part产 however, mouth suction in any form has beeno eliminated by the purchase of additional auto matic sampling equipment or combined samplers. and diluters.

\section{AutoAnalyzer Maintenance Procedures and the Disposal of Effluent

The report of the PHLS Working Party (1968) drew attention to the dialysis unit of the Auto $=$ Analyzer continuous flow system, and recom $\vec{\omega}$ mended that the membranes should not be changed with ungloved hands. Our precaution\$s here are more detailed, since this unit of the AutoAnalyzer system tends to get progressivelye blocked with a residue of protein material. As the dialyser normally operates at $37^{\circ} \mathrm{C}$, this could well provide a particularly dangerous reservoip of the hepatitis virus.

We did consider attempting to sterilize then dialyser unit by heat before changing the meme brane, but were concerned about the possibility of damaging the expensive plastic plates between which the semipermeable membrane is held $\vec{v}$ The procedure which we have adopted has beens first to pump $2 \%$ glutaraldehyde for $30 \mathrm{~min}$ through the dialyser unit. Then, wearing gloveso the technician places the dialyser unit on a trays and takes it to a fume cupboard hood which has been set aside for the changing of membranes $\frac{0}{D}$ The unit is dismantled under the hood, the technician at this time wearing surgical rubbero gloves (ie, not the gloves chosen for genera $\vec{P}$ laboratory use) and the parts are then placed in $2 \%$ glutaraldehyde and left there for 30 minutes: Glutaraldehyde at this concentration does not damage the unit, but still has a significant disinfecting action. Concentrated glutaraldehyde? is added to the dialyser bath to give a fina: concentration of $2 \%$ and kept for 30 minutes at $37^{\circ} \mathrm{C}$. The water in the bath is then replaced ando the dialyser components are washed thoroughly in water before assembling a new dialyser unit. 음

When disposing of samples from AutoAnalyzer sample plates, staff wear gloves. Each uncappeder cup is handled separately with steel forceps $N$ submerged in $10 \%$ Chloros, and the forceps ares flamed immediately the plate has been emptied. 0 The cups are left in Chloros overnight and then wearing gloves, the technician pours off the Chloros and disposes of the cups into red plastices bags (see below). Any cups which may have been capped, for example, for possible use in within-ō batch or between-batch repeat analyses (as parti of quality control procedures), are disposed of directly into the plastic bags if they have not
after all been used again.

The plates or rings used for holding samplesô analysed on AutoAnalyzers are soaked after use by standing overnight in $10 \%$ Chloros. They areō 
then rinsed with water and dried before being used again.

The effluent from AutoAnalyzers runs directly into channels and drains serving the individual benches; the detailed construction of these makes it impracticable to have the waste lines dripping initially into containers with disinfectant, as recommended by the PHLS Working Party (1968). At the end of the day these channels are washed down with $10 \%$ Chloros. Sinks in the department are similarly washed down in the evening if any biological material has been disposed of.

\section{Other Arrangements for Disposal and Disinfection}

Biological material that is to be destroyed in the hospital incinerator is, in general, transported there in heavy duty red plastic bags. These have absorbent material placed inside them before sample tubes or AutoAnalyzer cups, for instance, are discarded into them. Any containers that have been soaked in Chloros before disposal have most of this decanted before the containers are disposed of. Urine samples are handled differently since their containers are not readily disposable; with these, $50 \mathrm{ml}$ concentrated Chloros is added to each Winchester and the contents left to stand overnight before discarding the urine carefully down the sluice and washing out the bottle. Faecal specimens are transported in their cartons under leak-proof conditions to the incinerator.

Pasteur pipettes represent one item of equipment referred to in these precautions where the practice is still to wash and reuse, as long as they are fit to be used again. After standing overnight in $10 \%$ Chloros, the disinfectant is decanted, the pipettes are washed, dried in a hot air oven $\left(100^{\circ} \mathrm{C}\right)$, and used again.

\section{Miscellaneous Points}

A special telephone has been installed in the reception area, where most enquiries about laboratory work are received. Staff can use this telephone by depressing a series of buttons using the elbow and do not need to lift a receiver, nor do they need to remove their gloves or wash their hands rapidly in response to the telephone's unscheduled interruptions of their work.

Before this hepatitis outbreak, in common with many other chemistry laboratories, we used to prepare pools of serum or plasma from the unused portions of samples submitted from patients. This practice has been stopped, and the use of specimens from patients for quality control purposes is now restricted to repeating the analysis of limited numbers of these samples to provide data about within-batch and betweenbatch performance. We have made enquiries about the possibility of using ionizing radiation $\frac{\varrho}{\overline{5}}$ to render samples of blood safe-either for ${ }_{0}$ subsequent use as part of quality control pro-s cedures or, preferably, safe before any form ofo analytical work. So far this line of enquiry has: appeared impracticable, at least for large-scales? use, and its further consideration also awaitso the development of methods to prove that the hepatitis virus has been inactivated. Ionizing $\frac{}{5}$. radiation would appear to be inappropriate for specimens on which serum enzyme determinationso are to be carried out.

Special attention needs to be drawn to the $\overrightarrow{0}$ Astrup or similar types of equipment widely used $\vec{\omega}$ for the measurement of blood $p \mathrm{H}, \mathrm{PCO}_{2}$, ando other acid-base indices. For those determinations requiring tonometry, it is important to cover thein tonometer while the samples are equilibrating to ${ }_{0}^{\omega}$ prevent droplets of blood being disseminated through the air. Also, special care needs to be $\mathrm{G}$ taken so as to avoid puncturing gloved hands은 when manipulating capillary sample tubes andin the disposal of syringe needles.

Details of the recurrent expenditure attributable to these precautions, together with information about the suppliers of the less well knownळ items, are given in the Appendix. Costs are totalling about $£ 1,600$ for a full year. To give some idea of perspective, when translating these ${ }^{\circ}$ costs to other departments possibly contemplat-O ing adopting similar precautions, the component of the floor area in this laboratory devoted to theo diagnostic work on specimens from patientso totals approximately $400 \mathrm{sq} \mathrm{m}$ and there are $\propto$ 35 members of technical staff.

The PHLS Working Party (1968) suggested that the regular screening of staff by the measurement of alanine aminotransferase (GPT) activityọ might be of value in helping with the early detection of cases in dialysis units. We have? decided not to adopt this procedure for our staff, partly because there is no definitive means of halting or modifying the course of serum hepatitis once it has been contracted, and partly because this might cause more disturbance to the staff? than the low incidence of serum hepatitis among $\frac{}{0}$ laboratory workers would appear to warrant. This decision may require reconsideration, but if $N$ laboratory staff is to be screened earlier indications of infection with the virus of serum hepatitis would probably be provided by testing ${ }_{\sigma}^{\omega}$ for Australia antigen (Krugman and Giles, 1970).

\section{Discussion}

The main criticism that can be levelled at the code of practice drawn up for this department? and described in this paper is the absence of anyo proof that the precautions are effective. Many of the precautions were introduced in May 1970 
but further changes in procedure were held up pending the outcome of the detailed review of laboratory work. Apart from the decisions about changes in procedure that were reached quickly, others required much thought and discussion, and the introduction of the full scheme was delayed until decisions had been reached. It was felt that the piecemeal and progressive introduction of additional precautions during the period of the review could only have adverse effects on the morale of staff. The effectiveness of these precautions, and the demonstration of shortcomings in particular areas of work, will depend for their definition upon the development of methods of isolating, culturing, and identifying the virus of serum hepatitis.

We do know that a member of staff in this department contracted serum hepatitis in July, before the full range of precautions was in operation. This technician has since recovered fully, and a careful retrospective epidemiological enquiry has failed to establish any history of an 'accident situation'. In this technician's case, the two areas of potential risk considered to have been most likely to be responsible were the formation of aerosols at the time of centrifuging blood specimens or at the time of equilibrating blood samples in the Astrup apparatus.

We attach great importance to the appointment of a safety officer, with arrangements for deputies. We would also stress the importance of taking the staff into the confidence of the head of the department, as otherwise the fear and uncertainty engendered by an outbreak of serum hepatitis can have very adverse effects on morale, causing distraction from work while rumours are discussed, and even leading to resignations. These undesirable effects have occurred to a minor degree with the present outbreak of serum hepatitis, but they could very easily have assumed much more serious proportions.

We wish to thank Professor B. P. Marmion and many other members of staff in the Department of Bacteriology for their interest and advice.

\section{References}

Allen, J. C., and Kunkel, H. G. (1966). Antibodies against $\gamma$-globulin after repeated blood transfusions in man. $J$. clin. Invest., 45, 29-39.

Krugman, S., and Giles, J. P. (1970). Viral hepatitis: new light on an old disease. J. Amer. med. Ass., 212, 1019-1029.

Lancet (1970). Leading article. More about Australia antigen and hepatitis. Lancet, 2, 347-349.

New England Journal of Medicine (1970). Leading article. Control of hepatitis on hemodialysis units. New Engl. J. Med., $282,1488$.

Nyström, B., and Ringertz, O. (1967). Hepatitsymposiet. Epidemiologiska erfarenheter. Lakartidningen, 64, 1427-1435.

Public Health Laboratory Service: Report of a Working Party (1968). Infection risks of haemodialysis-some preventive aspects. Brit. med. J., 3, 454-460.

Vyas, G. N., Perkins, H. A., and Fudenberg, H. H. (1968). Anaphylactoid transfusion reactions associated with anti-IgA. Lancet, 2, 312-315.

\section{Appendix}

\begin{tabular}{|c|c|c|}
\hline Article & Unit Cost & $\begin{array}{l}\text { Six } \\
\text { Months } \\
\text { Expendi } \\
\text { ture }\end{array}$ \\
\hline
\end{tabular}

Gloves

Regent surgeons' gloves

(L.R. Industries Ltd,

Hall Lane, Chingford,

London, E4)

b Disposaglove

(Arbrook Products

Bankhead Avenue,

Sighthill, Edinburgh 11)

Chloros: $10 \%$ solution

(Scottish Agricultural

Industries Ltd, 38 Queen

Charlotte Street, Leith, Edinburgh 6)

Cidex ( $2 \%$ glutaraldehyde)

solution

(Ethicon Ltd,

Sighthill Industrial Estate, Edinburgh 11)

Benchkote

(H. Reeve Angel Ltd,

14 New Bridge Street

London EC4V 6AY)

Aprons 'Atholl Crescent'

lightweight

$£ 3.10$ s per box of 36360

0

(Hewats of Edinburgh,

Teviot Place, Edinburgh

EHI 2RA)

Phisohex

(Bayer Products Ltd,

Surbiton-on-Thames)

Savlon barrier cream (ICI Ltd)

Cellulose wadding (absorbent)

Cellosene (Robinsons of

Chesterfield, Wheatbridge

Mill, Chesterfield)

Atrixo hand cream

(Smith \& Nephew,

Bessimer Road, Welwyn

Garden City)

Sterzac soap

(Macarthys Ltd, 7/11

Glenpannar Road, Glasgow)

Masks Aseptex No. 1800

(The 3M Company, Medical

Products Division, 3M

House, Corporation Street,

Manchester 4)

Heavy duty plastic bags (Goggins Ltd, Dixon's

Blazes, 562 Lawmoor Street, Glasgow C5)

Loudspeaking telephone $3 \quad$ Rental charge $f 4$ GPO DLE 511 per quarter

$£ 3.5$ s per ree

( 50 in. rolls)

$£ 3.10$ s per dozen

1710

$£ 6.13 .4 \mathrm{~d}$ per gallon

$£ 1.5$ s per dozen tubes

$£ 2.5 .10 \mathrm{~d}$

15 s per dozen

$11 \mathrm{~d}$ each

$£ 3.8 .6 \mathrm{~d}$

Total expenditure

$£ 4.11$ s per 100

80

Table Costs from 1 May to 31 October 1970 